\section{Childhood adversity and certain mental health disorders are associated with increased risk of incident drug use among adults}

\section{QUESTION}

Question: Are sociodemographic variables, childhood adversity or mental health disorders associated with incident drug use in adults who report no prior drug use?

People: 26935 community dwelling adults enrolled in the National Epidemiological Survey on Alcohol and Related Conditions. Participants had to report no history of illicit drug use during the first wave of the study (from 2001 to 2002) for inclusion in the current analysis. Incident drug use was assessed in a second wave of interviews (from 2004 to 2005).

Setting: Community setting, USA; from 2001 to 2005.

Risk factors: Sociodemographic variables: income, race, education, marital status, age, sex and region. Childhood traumatic events: physical abuse, witnessing violence in the home, neglect and sexual assault. Family history of substance use: first-degree relatives (siblings or parents) with a history of problem drinking or problem drug use. Mental health disorders (defined using DSM-IV criteria): any mood disorder (major depression, dysthymia, bipolar I, bipolar II), any anxiety disorder (panic disorder, social phobia, specific phobia, posttraumatic stress disorder, generalised anxiety disorder), any personality disorder (schizoid, schizotypal, paranoid, antisocial, borderline, narcissistic, histrionic, obsessive-compulsive, avoidant, dependent), any alcohol use disorder (alcohol abuse and dependence) and nicotine dependence.

Outcomes: First time illicit drug use (sedatives, tranquilisers, opioids, amphetamines, cannabis, cocaine, hallucinogens, inhalants, heroin and other).

\section{METHODS \\ Design: Prospective cohort study. \\ Follow-up period: Three years.}

\section{MAIN RESULTS}

Of the 26935 participants who reported no history of drug use at baseline 1145 (4.3\%) used illicit drugs for the first time during follow-up. The most commonly used substances were cannabis $(44.4 \%)$, opioids $(39.6 \%$, excluding heroin) and sedatives $(25.5 \%)$. A number of sociodemographic variables were associated with decreased odds of incident drug use (female sex; increasing age; Asian, Hawaiian and Hispanic ethnicity; being married). After adjustment for sociodemographic variables, all measured childhood trauma variables were associated with an increased odds of reporting incident illicit drug use: physical abuse by parent/ guardian (OR 2.74, 95\% CI 1.93 to 3.88), physical abuse by anyone else (OR $2.05,95 \%$ CI 1.49 to 2.82 ), witnessed fights at home (OR 2.12, 95\% CI 1.70 to 2.66), neglect by parent/guardian (OR 2.25, 95\% CI 1.55 to 3.25 ) and sexual assault (OR $2.55,95 \%$ CI 2.00 to 3.26 ). Having a family history of substance use disorders was also associated with an increased risk of incident drug abuse (first-degree relative with alcohol problem: OR 1.49, 95\% CI 1.27 to 1.74 ; firstdegree relative with drug problem: OR 1.40, 95\% CI 1.12 to 1.76). After adjusting for sociodemographic variables, childhood trauma and family history of substance use the following mental health disorders were associated with an increased odds of reported incident drug use: any mood disorder (OR 1.31, 95\% CI 1.04 to 1.64), any personality disor$\operatorname{der}(\mathrm{OR} 1.82,9 \%$ CI 1.50 to 2.20 ), nicotine dependence (OR $1.41,95 \%$ CI 1.09 to 1.83 ) and any alcohol use disorder (OR $1.96,95 \%$ CI 1.48 to 2.60$)$. The diagnosis of any anxiety disorder was not significantly associated with incident drug use (OR $0.85,95 \%$ CI 0.64 to 1.13 ).

\section{CONCLUSIONS}

Childhood trauma, substance use problems among family members, nicotine dependence and a diagnosis of any mood, personality or alcohol use disorder are associated with increased odds of incident drug use among abstaining community dwelling adults.

\section{ABSTRACTED FROM \\ Harrington M, Robinson J, Bolton SL, et al. A longitudinal study of risk factors for incident drug use in adults: findings from a representative sample of the US population. Can J Psychiatry 2011;56:686-95.}

Correspondence to: Dr J M Bolton, PZ430-771 Bannatyne Avenue, Winnipeg MB R3E 3N4, Canada; jbolton@exchange.hsc.mb.ca

Sources of funding: Manitoba Health Research Council, Canadian Institutes of Health Research.
$\mathrm{H}$ arrington et al conducted an interesting and innovative epidemiologic analysis of risk factors for incident drug use in adults in the US general population with state-of-the art measures of psychiatric disorders, illegal drug use, childhood adversity and family history. Their manuscript fits well with previous research conducted in this area. ${ }^{1}$ The authors found that $4.3 \%$ of those who had never used drugs in first wave of the study went on to first use an illegal drug within a 3-year time period. As expected, pre-existing mental disorders and personality disorders were associated with incident drug use. Family history of alcohol or drug problems and having experienced childhood adversity were associated with incident drug use. However, since childhood traumatic events were measured not when respondents were children, but when they were already adults, these measures might be subject to recall bias. Moreover, family history measures were rather vague and were obtained from the respondent, and not from their relatives. It would be interesting if the authors had taken full advantage of the dataset by comparing these adult incident drug users to those respondents who had already initiated drug use before adulthood, in regards to the correlates included in this study. After reading the paper, one wonders what truly differentiates them from respondents who initiated drug use when they were younger, that is, in adolescence, is it only the opportunity to first use a specific drug, or are other factors involved? ${ }^{2}$ Nevertheless, this is an important contribution to the literature on the epidemiology of drug use and the findings are consistent with previous studies.

\section{Silvia S Martins}

Department of Mental Health, Johns Hopkins Bloomberg School of Public Health, Baltimore, Maryland, USA

Competing interests None.

\section{REFERENCES}

1. Grant BF, Goldstein RB, Chou SP, et al. Sociodemographic and psychopathologic predictors of first incidence of DSM-IV substance use, mood and anxiety disorders: results from the Wave 2 National Epidemiologic Survey on Alcohol and Related Conditions. Mol Psychiatry 2009;14:1051-66.

2. Wagner FA, Anthony JC. Into the world of illegal drug use: exposure opportunity and other mechanisms linking the use of alcohol, tobacco, marijuana, and cocaine. Am J Epidemiol 2002:155:918-25. 